\title{
Nanocompósitos de Polietileno/Argila Bentonita Nacional: Influência da Argila e do Agente Compatibilizante PE-g-MA nas Propriedades Mecânicas e de Inflamabilidade
}

\author{
Gustavo F. Brito, Amanda D. de Oliveira, Edcleide M. Araújo, Tomás J. A. de Melo \\ Departamento de Engenharia de Materiais, UFCG
}

Renata Barbosa

Departamento de Engenharia de Processos, UFCG

\section{Edson N. Ito \\ Departamento de Engenharia de Materiais, UFSCar}

\begin{abstract}
Resumo: Nanocompósitos de polietileno de alta densidade/argila bentonita nacional foram preparados por intercalação por fusão. A influência da adição da argila sem tratamento e da argila tratada com o sal quaternário de amônio Cetremide e do polietileno graftizado com anidrido maléico (PE-g-MA) como agente compatibilizante foi avaliada. Os sistemas obtidos foram caracterizados por inflamabilidade, propriedades mecânicas e reológicas, difração de raios X e microscopia eletrônica de transmissão. Os resultados de difração de raios X e microscopia eletrônica de transmissão indicaram a obtenção de nanocompósitos com estruturas parcialmente esfoliadas e/ou intercaladas que conduziram ao aumento do módulo de elasticidade e do valor do torque. $O$ teste de inflamabilidade mostrou que a adição da argila na matriz polimérica e a presença do PE-g-MA retardaram a velocidade de queima do sistema, ou seja, diminuíram a inflamabilidade do PE puro. Em geral, o sistema contendo o agente compatibilizante (PE-g-MA) exibiu melhorias na estabilidade térmica e nanocompósitos parcialmente esfoliados foram formados, quando comparados à matriz de polietileno de alta densidade. O polietileno graftizado com anidrido maléico não apenas promoveu a esfoliação da argila, mas também facilitou a adesão carga-matriz, obtendo-se com isso melhorias nos resultados mecânicos do sistema.
\end{abstract}

Palavras-chave: Nanocompósitos, polietileno, argila bentonita nacional, inflamabilidade.

Polyethylene/National Bentonite Clay Nanocomposites: Influence of clay and PE-g-MA Coupling Agent on the Mechanical Properties and Flammability

Abstract: High density polyethylene/national bentonite clay nanocomposites were prepared with the melt intercalation technique. The effect of the clay without treatment and clay treated with quaternary ammonium salt Cetremide with polyethylene-graft-maleic anhydride as coupling agent was evaluated. The systems were characterized by flammability, mechanical and rheological properties, $\mathrm{X}$ ray diffraction and transmission electron microscopy. In general, the results of $\mathrm{X}$ ray diffraction and transmission electron microscopy showed partially exfoliated and/or intercalated structures with higher tensile modulus and torque value. The flammability test showed that the addition of clay in the polymer matrix and PE-g-MA reduced the burning rate, indicating an improved flammability resistance of the nanocomposites. In general, the system containing PE-g-MA coupling agent led to partially exfoliated nanocomposites with higher thermal stability than the high density polyethylene matrix. The polyethylene-graft-maleic anhydride promoted the exfoliation of clay and adhesion between matrix and filler, thus resulting in enhanced mechanical properties.

Keywords: Nanocomposites, polyethylene, national bentonite clay, flammability.

\section{Introdução}

O desenvolvimento da nanotecnologia nas últimas décadas conduziu a um crescimento e um grande interesse pela área de nanocompósitos devido às propriedades especiais apresentadas por estes materiais, não apenas por eles possibilitarem à obtenção das propriedades equivalentes a dos compósitos tradicionais, mas também por exibirem propriedades ópticas, elétricas, de barreira, de inflamabilidade e magnéticas únicas ${ }^{[1]}$. Recentemente, muita atenção vem sendo dada aos nanocompósitos poliméricos; especialmente os nanocompósitos desenvolvidos com silicatos em camadas, que representam uma alternativa aos compósitos desenvolvidos com cargas convencionais e utilizam níveis mínimos $(<10 \%)$ de nanocargas denominadas argilas

Autor para correspondência: Edcleide M. Araújo, Departamento de Engenharia de Materiais, UFCG, Rua Aprígio Veloso 882 - Bodocongó,

CEP: 58109-970, Campina Grande, PB, Brasil.E-mail: edcleide@dema.ufcg.edu.br 
organofílicas $^{[2-4]}$. Essa nova classe de materiais é definida por Komarneni (1992) $)^{[5]}$, em sua ampla revisão sobre o assunto, como sendo nanocompósitos contendo mais de uma fase sólida (amorfa, semicristalina, cristalina ou combinação delas) e apresentando pelo menos uma com dimensão em tamanho nanométrico.

Silicatos como a montmorilonita (MMT), argilomineral predominante na argila bentonita, têm recebido grande atenção nas ultimas décadas, como materiais reforçantes para polímeros, devido à alta razão de aspecto característica destes materiais e à possibilidade de intercalação/esfoliação das camadas do silicato na matriz polimérica. Tais argilominerais têm uma camada estrutural, tipicamente com $1 \mathrm{~nm}$ de espessura, que se propriamente esfoliada pode levar a produção de uma matriz polimérica com grande número de partículas finamente dispersas (aproximadamente $1 \mu \mathrm{m}$ de dimensões laterais). Geralmente, para permitir uma melhor interação da argila inorgânica com polímeros orgânicos, a mesma deve ser transformada em organofílica ${ }^{[6-8]}$. As argilas organofílicas têm sido obtidas a partir da adição de sais quaternários de amônio (com pelo menos 12 ou mais átomos de carbono em sua cadeia) em dispersões aquosas de argilas esmectíticas sódicas. Nessas dispersões aquosas as partículas elementares ou lamelas, devem encontrar-se (em maior ou menor grau) umas separadas das outras (e não empilhadas), facilitando a introdução dos compostos orgânicos, que irão torná-las organofílicas. Dessa forma, os cátions orgânicos do sal substituem os cátions da argila sódica, passando-a de hidrofílica para organofílica ${ }^{[8-17]}$.

Quando as argilas organofílicas são incorporadas ao polímero, o caráter de reforço que as partículas da argila exercem deve-se às restrições da mobilidade das cadeias poliméricas em contato com as partículas da argila. Desta forma, a melhoria nas propriedades nos nanocompósitos tais como: resistência à tração, compressão, fratura e os aumentos do módulo de Young têm sido relacionados com a dispersão; o grau de delaminação; o fator de forma da argila; e as interações interfaciais polímero-argila ${ }^{[18]}$. Como já mencionado, as propriedades térmicas, óticas, de inflamabilidade e de resistência a solventes também são melhoradas pela incorporação da argila ao polímero. A maior estabilidade térmica está relacionada com a diminuição da difusão das moléculas de oxigênio para o interior do nanocompósito devido à barreira formada pelas partículas da argila. Assim, sem o oxigênio, principal fator na deteriorização do polímero, o nanocompósito é mais resistente à degradação oxidativa ${ }^{[16,19]}$. Este trabalho tem como objetivo analisar a influência da adição de uma argila bentonita nacional e do compatibilizante polietileno graftizado com anidrido maléico (PE-g-MA) nas propriedades morfológicas, de inflamabilidade, mecânicas e reológicas de nanocompósitos de polietileno de alta densidade moldados em injetora de bancada.

\section{Experimental}

Este trabalho utilizou como matrizes poliméricas o polietileno de alta densidade (JV-060U), IF = 6,1-8,0 g/10 $\mathrm{min}^{[20]}$, fornecido pela Braskem/BA e o polietileno graftizado com $1 \%$ de anidrido maléico (PB3009), IF = 5,0 g/10 min, fornecido pela Crompton/SP. A argila utilizada foi a BRASGEL PA (sódica ativada), passada em peneira ABNT $n^{\circ} 200$ $(\mathrm{d}=0,074 \mathrm{~mm})$, de cor creme, fornecida pela Indústria Bentonit União Nordeste (BUN), localizada na cidade de Campina Grande - PB. A capacidade de troca de cátions (CTC) dessa argila é de $90 \mathrm{meq} / 100 \mathrm{~g}$ (método de adsorção de azul de metileno). Para tornar a argila compatível com o polímero, os íons de sódio presentes entre as lamelas da argila são trocados por íons de amônio para produzir a argila organofílica. Essa troca foi realizada na presença do sal quaternário de amônio Cetremide (brometo de hexadeciltrimetil amônio), produzido pela VETEC/SP. Para a obtenção da argila organofílica foi realizado tratamento apropriado para o sal Cetremide, baseando-se na CTC da argila, conforme trabalhos já reportados pelo grupo ${ }^{[12,14,15,17]}$.

Foram obtidos nanocompósitos com PE e PE-g-MA com 3 e $5 \%$ em peso de argila. Para efeito de comparação nos nanocompósitos obtidos, foi analisada a influência da argila sem tratamento (AST), com e sem a presença do sal Cetremide, variando-se o teor de sal em 1 e 3\%, bem como, foi produzido um híbrido de PE com $3 \%$ em peso de argila tratada (ACT) com o sal Cetremide e 1\% de PE-g-MA, conforme Tabela 1. Isto foi realizado com o objetivo de avaliar a influência da argila e do sal misturados simultaneamente com a matriz polimérica de $\mathrm{PE}$ em equipamento de mistura e também para avaliar o efeito da argila obtida por tratamento via troca de cátions (argila organofílica) e do compatibilizante PE-g-MA em nanocompósito de PE. O PE-g-MA foi utilizado com o intuito de melhorar a interação do PE com a argila, já que o grupo funcional MA favorece à polaridade não encontrada no PE que é apolar. A argila foi incorporada nas matrizes poliméricas em um Reômetro de Torque System 90 da Haake. As condições de processamento utilizadas foram: $190{ }^{\circ} \mathrm{C}$ e $60 \mathrm{rpm}$ durante 10 minutos. O material obtido foi granulado e em seguida moldado por injeção em injetora de bancada, modelo Ray Ran, na temperatura de $210{ }^{\circ} \mathrm{C}$ sob pressão de $100 \mathrm{psi}$, na forma de corpos de prova de inflamabilidade (UL 94HB), tração (ASTM D638) e impacto (ASTM D256).

Difração de raios X (DRX): As análises de DRX foram conduzidas em aparelho XRD-6000 Shimadzu, utilizando-se radiação $\mathrm{K} \alpha$ do cobre, tensão de $40 \mathrm{kV}$, corrente de $30 \mathrm{~mA}$, varredura entre $2 \theta$ de 2 a $30^{\circ}$ e velocidade de varredura de $2 \%$ min.

Microscopia Eletrônica de Transmissão (MET): O microscópio eletrônico de transmissão utilizado foi o da marca PHILIPS CM 120, operando a uma voltagem de aceleração de $120 \mathrm{kV}$. As amostras foram retiradas do centro do corpo de prova de impacto e preparadas através da redução de área pelo procedimento do "trimming". Os cortes das amostras foram realizados em um ultramicrótomo da marca RMC modelo MT-7000 usando-se uma faca de diamante da marca Diatome tipo Cryohisto $45^{\circ}$, em condições criogênicas. 
Tabela 1. Composições utilizadas nos sistemas.

\begin{tabular}{lccccc}
\hline \multicolumn{1}{c}{ Composição } & $\begin{array}{c}\text { PE } \\
(\boldsymbol{\%})\end{array}$ & $\begin{array}{c}\text { PE-g-MA } \\
(\boldsymbol{\%})\end{array}$ & $\begin{array}{c}\text { Argila sem tratamento } \\
(\text { AST })(\boldsymbol{\%})\end{array}$ & $\begin{array}{c}\text { Argila com tratamento } \\
(\text { ACT })(\boldsymbol{\%})\end{array}$ & $\begin{array}{c}\text { Sal cetremide } \\
(\%)\end{array}$ \\
\hline PE puro & 100 & - & - & - & - \\
PE-g-MA puro & - & 100 & - & - & - \\
PE/AST & 95 & - & 5 & - & - \\
PE-g-MA/AST & - & 95 & 5 & - & - \\
PE/AST/Cetremide 1\% & 94 & - & 5 & - & 1 \\
PE/AST/Cetremide 3\% & 92 & - & 5 & - & 3 \\
PE-g-MA/AST/Cetremide 1\% & - & 94 & 5 & - & 1 \\
PE-g-MA/AST/Cetremide 3\% & - & 92 & 5 & - & - \\
PE/ACT & 97 & - & - & 3 & - \\
PE-g-MA/ACT & - & 97 & - & 3 & - \\
PE/PE-g-MA/ACT & 96 & 1 & - & 3 & - \\
\hline
\end{tabular}

Caracterização Reológica: para a caracterização reológica, os sistemas foram obtidos em misturador interno acoplado a um Reômetro de Torque System 90 da Haake, com temperatura de $190{ }^{\circ} \mathrm{C}$ e velocidade $60 \mathrm{rpm}$, por um tempo de 7 minutos.

Teste de Inflamabilidade horizontal, UL $94 \mathrm{HB}^{[21]}$ : O ensaio é aplicado para materiais que continuam a queimar e propagam a chama, após a retirada da chama inicial. É utilizado com o objetivo de classificar como $94 \mathrm{HB}$ o material polimérico como uma velocidade de queima abaixo de um valor mínimo especificado ${ }^{[14,16,17]}$.

Propriedades Mecânicas: Para o ensaio de tração, foi utilizada uma máquina universal de ensaios Lloyd Instruments $10 \mathrm{KN}$, operando a uma velocidade de deformação de $50 \mathrm{~mm} / \mathrm{min}$ para obtenção do módulo de elasticidade, resistência no escoamento e alongamento na ruptura, conforme norma ASTM D638. Para o ensaio de impacto, os corpos de prova foram entalhados com entalhe de profundidade aproximadamente $2,5 \mathrm{~mm}$ em entalhador Ceast, segundo norma ASTM D256. Eles foram então submetidos ao impacto em um equipamento Ceast, modelo Resil $5.5 \mathrm{~J}$, com pêndulo de 2,75 $\mathrm{J}$ acoplado a um sistema de aquisição de dados, via software.

\section{Resultados e Discussão}

Neste estudo, foram obtidos nanocompósitos de PE com $5 \%$ em peso de argila sem tratamento (AST), sem o sal quaternário de amônio Cetremide e na presença do sal (1 e 3\%). Um híbrido também foi obtido com $3 \%$ em peso de argila tratada (ACT) com o mesmo sal. O objetivo desse estudo foi verificar a influência da adição da argila e do compatibilizante PE-g-MA no desempenho de nanocompósitos de PEAD.

\section{Caracterização estrutural}

Para investigar a estrutura dos nanocompósitos produzidos foram utilizadas as técnicas de DRX e MET, já que essas são ferramentas essenciais indispensáveis para avaliar a intercalação/esfoliação dos nanocompósitos. A Figura 1 apresenta os difratogramas da argila sem tratamento (AST), dos sistemas PE e PE-g-MA com 5\% de AST e 1 e 3\% do sal Cetremide. Para os sistemas PE/AST e PE-g-MA/AST/Cetremide

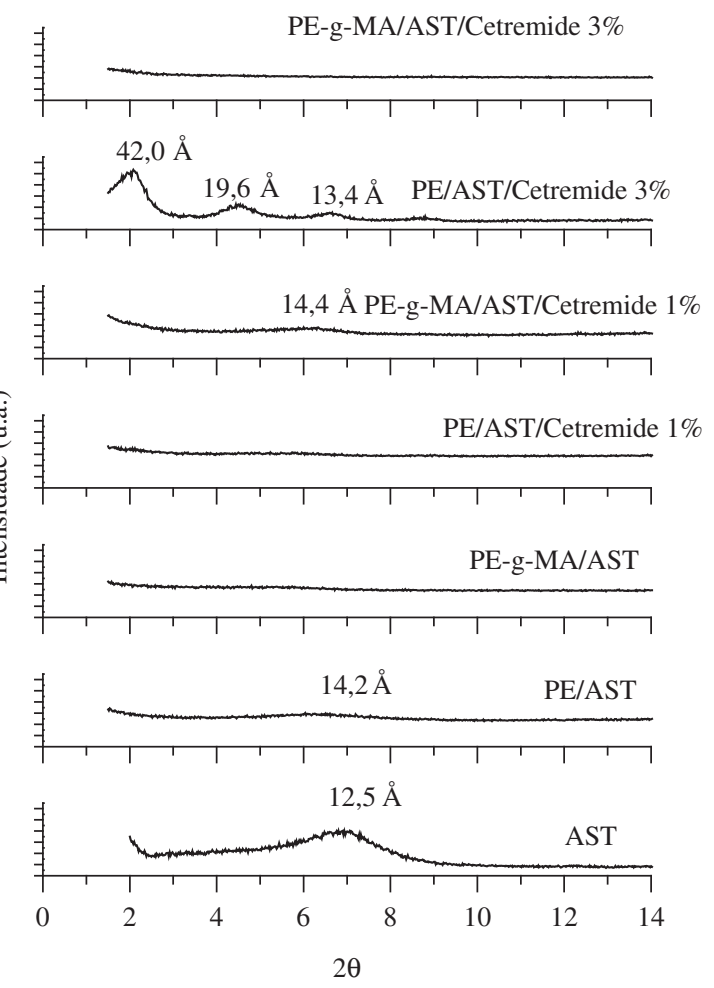

Figura 1. Difratogramas da argila sem tratamento (AST) e dos sistemas PE e PE-g-MA com 5\% de AST e 1 e $3 \%$ de Cetremide.

$1 \%$ observa-se a presença de um ombro correspondente à distância basal $\left(\mathrm{d}_{001}\right) 14,2 \AA$ A e um deslocamento para $2 \theta=6,2^{\circ} \mathrm{e}$ $\mathrm{d}_{001}=14,4 \AA$ e $2 \theta=6,1^{\circ}$, respectivamente, que são próximos da posição do pico obtido para a argila sem tratamento $\left(\mathrm{d}_{001}=12,5 \AA\right.$ e $\left.2 \theta=7,1^{\circ}\right)$, evidenciando um pequeno aumento da distância basal entre os planos $\mathrm{d}_{001}$, ou seja, pode ter acontecido uma intercalação de algumas cadeias de PE entre as camadas da argila. As composições PE-g-MA/AST, $\mathrm{PE} / \mathrm{AST} / \mathrm{Cetremide} 1 \%$ e PE-g-MA/AST/Cetremide 3\% não apresentaram pico basal o que sugere esfoliação da argila. A composição PE/AST/Cetremide 3\% apresenta um pico com uma distância basal de $42 \AA$ e deslocamento $2 \theta$ para $2,1^{\circ}$, correspondente à intercalação das moléculas do polietileno entre as camadas da argila sem tratamento; a presença de um ombro correspondente à distância $19,6 \AA\left(2 \theta=4,5^{\circ}\right)$ que 
pode indicar que uma pequena parte das camadas da argila sem tratamento foi intercalada pelas moléculas do polímero e um segundo ombro (13,4 $⿱$ A). Segundo Zanetti e Costa ${ }^{[22]}$, a presença do ombro indica um aumento da distância basal devido à intercalação do polímero e o pico indica que foi mantida a regularidade do espaçamento interlamelar de algumas camadas da argila. Essas estruturas também foram observadas por Barbosa et al. ${ }^{[20]}$ e Paiva et al. ${ }^{[23]}$.

As fotomicrografias da Figura 2 podem esclarecer e confirmar as estruturas obtidas nesses difratogramas. Conforme observado para os sistemas PE/AST (Figura 1 e Figura 2a) e PE-g-MA/AST/Cetremide 1\% (Figura 1 e Figura 2e), há aglomerados de argila com comprimento maior que $\sim 200 \mathrm{~nm}$, evidenciando uma pequena intercalação das cadeias do polímero entre as lamelas da argila como reportado acima.



(a)

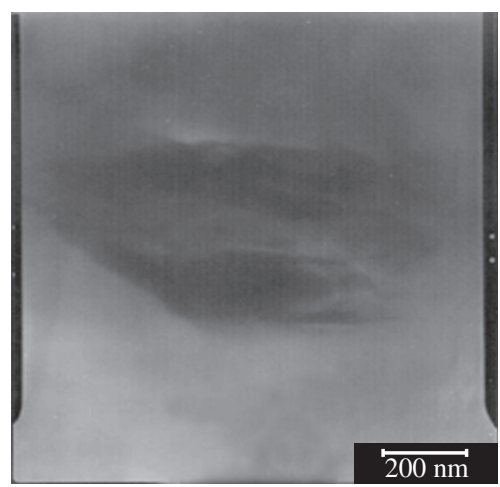

(b)

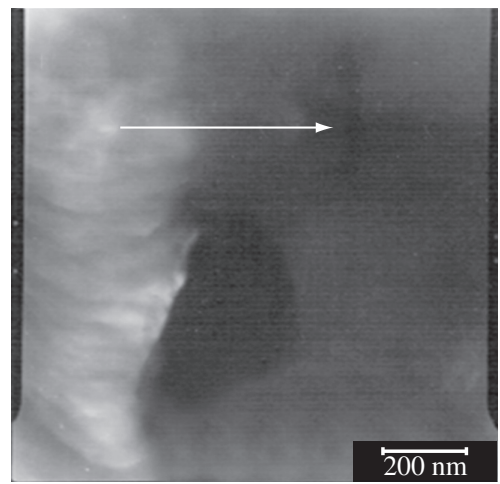

(c)
Como mencionado para os difratogramas com os sistemas PE-g-MA/AST, PE/AST/Cetremide $1 \%$ e PE-g-MA/AST/ Cetremide $3 \%$ que não apresentaram pico basal algum, as Figuras $2 b, 2 c$ e $2 \mathrm{f}$ apresentam as fotomicrografias desses sistemas. É interessante observar que a Figura $2 b$ apresenta um aglomerado de comprimento maior que $\sim 200 \mathrm{~nm}$ e, aparentemente menor do que aquele apresentado na Figura 2a. Na Figura $2 \mathrm{c}$ pode ser observado um aglomerado, mas aparecem também algumas lamelas empilhadas, como mostra a seta. E a Figura $2 \mathrm{f}$ mostra algumas lamelas de argila com tendência à esfoliação para o sistema PE-g-MA/AST/Cetremide 3\%, o que confirma o não aparecimento do pico no difratograma da Figura 1, e que pode também estar relacionado com o melhor comportamento de inflamabilidade apresentado por esse sistema. Muito provavelmente, o cisalhamento promovido pelo

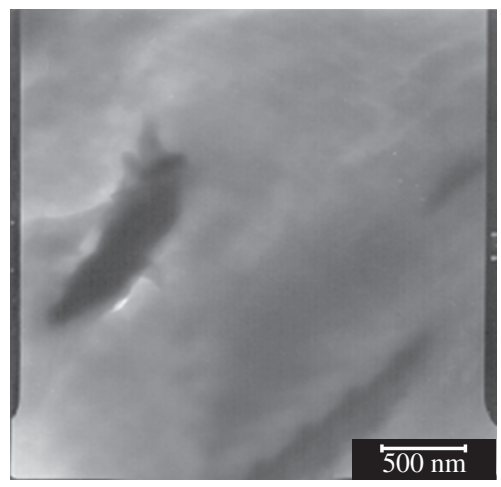

(d)

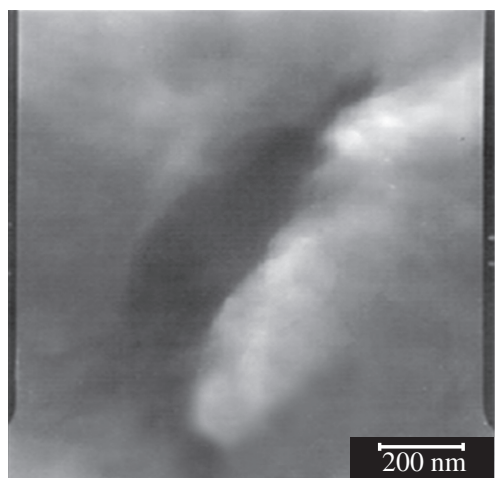

(e)

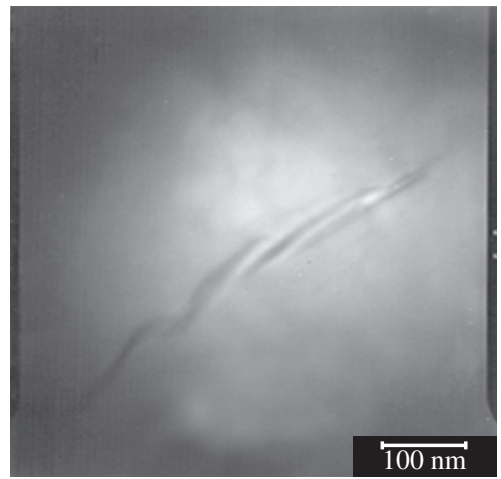

(f)

Figura 2. Fotomicrografias dos sistemas PE com 5\% em peso de argila sem tratamento: a) PE/AST; b) PE-g-MA/AST; c) PE/AST/Cetremide 1\%; d) PE/AST/Cetremide 3\%; e) PE-g-MA/AST/Cetremide 1\%; e f) PE-g-MA/AST/Cetremide $3 \%$. 
torque no equipamento de mistura não foi efetivo para separar completamente as lamelas da argila na matriz polimérica. Portanto, esses sistemas com argila sem tratamento apresentam estruturas intercaladas e/ou parcialmente esfoliadas.

A Figura 3 apresenta os difratogramas da argila com tratamento (ACT) e dos sistemas PE/ACT, PE-g-MA/ACT e PE/PE-g-MA/ACT com 3\% de ACT. A argila organofílica (ACT) apresenta um pico correspondente à distância basal $\left(\mathrm{d}_{001}\right)$ de $20,4 \AA$ e um deslocamento para $2 \theta=4,2^{\circ}$ que evidencia intercalação do sal quaternário de amônio entre as camadas da argila, comparando-se com a AST da Figura 1 que apresentou $\mathrm{d}_{001}=12,5 \AA$ e $2 \theta=7,1^{\circ}$. Observa-se também que a composição $\mathrm{PE} / \mathrm{ACT}$ apresentou um deslocamento do ângulo $2 \theta=2,38^{\circ} \mathrm{e}$ $\mathrm{d}_{001}=37,0 \AA$ em relação à ACT, correspondente a intercalação de moléculas de polietileno entre as lamelas da argila. Um segundo ombro aparece correspondente à distância 19,0 $\AA$ e $2 \theta=4,6^{\circ}$ que pode indicar que uma pequena parte das camadas da argila tratada não foi intercalada pelas moléculas de PE já que esses valores estão muito próximos da argila ACT. Para a composição PE-g-MA/ACT não foi observada a presença de picos indicando que a estrutura do nanocompósito obtido pode ter sido completamente esfoliada. O híbrido PE/PE-g-MA/ACT apresenta um aumento da distância basal para 42,3 $\AA$ e $2 \theta=2,16^{\circ}$, correspondente à intercalação de moléculas do PE nas camadas da argila modificada e a presença de um segundo ombro correspondente à distância $19,2 \AA$ e $2 \theta=4,72^{\circ}$ que pode indicar que uma pequena parte das camadas da argila modificada não foi intercalada pelas moléculas do polímero.

As fotomicrografias obtidas por MET das composições mencionados acima estão apresentadas na Figura 4. A Figura $4 \mathrm{a}$, correspondente à composição PE/ACT, mostra aglomerados grandes e pequenos de argila na matriz de PE, evidenciando a estrutura intercalada exibida no difratograma da Figura 3. Já a Figura 4b exibe um grande aglomerado, mas várias partículas de argila muito pequenas de comprimentos na faixa de $100 \mathrm{~nm}$ (mostradas por setas na figura), confirmando o desaparecimento do pico basal no difratograma da Figura 3 e evidenciando esfoliação parcial para essa composição. $\mathrm{Na}$ Figura 4c (PE/PE-g-MA/ACT) podem ser observadas diversas partículas de argila dispersas no PE com comprimentos na faixa de $500 \mathrm{~nm}$, confirmando a intercalação das moléculas de PE entre as lamelas da argila, bem como, as boas propriedades mecânicas e de inflamabilidade obtidas. É interessante enfatizar que mesmo utilizando uma argila organofílica (ACT) ou usando um sal quaternário de amônio simultaneamente com uma argila sem tratamento (AST) no PE é praticamente impossível se obter estruturas completamente esfoliadas devido provavelmente à natureza apolar do polímero usado. Por isso, foi usado também um PE-g-MA, de natureza polar pela presença do grupo anidrido maléico e com baixa concentração (apenas $1 \%$ no híbrido), a fim de se melhorar essa interação polímero-argila. Aparentemente, a interação foi melhorada e estruturas parcialmente esfoliadas e intercaladas foram obtidas. Estudos estão sendo realizados utilizando um equipamento com maior eficiência de mistura, no caso uma extrusora de

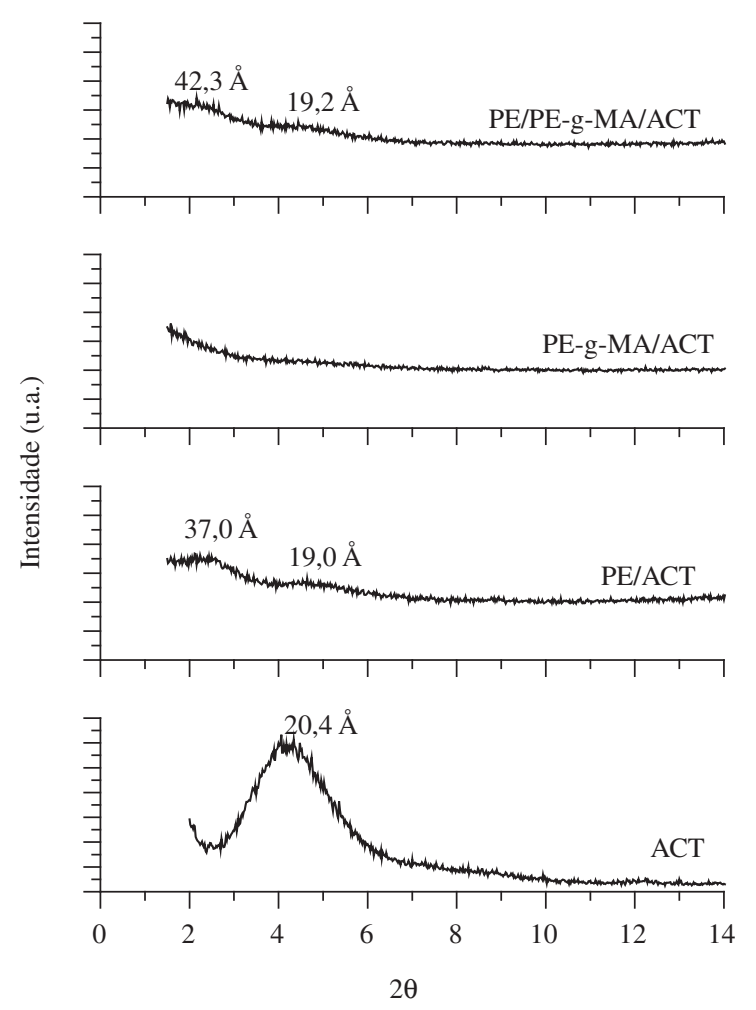

Figura 3. Difratogramas da argila com tratamento (ACT) e dos sistemas PE/ACT, PE-g-MA/ACT e PE/PE-g-MA/ACT com $3 \%$ de ACT.

dupla rosca, e com quantidades maiores de PE-g-MA (sempre observando a questão custo/benefício já que o PE-g-MA tem um custo bem superior comparado ao PE) com o objetivo de se obter estruturas mais bem esfoliadas e com melhoramentos mais significativos nas propriedades mecânicas e de inflamabilidade em relação ao PE puro.

\section{Teste de inflamabilidade horizontal, UL 94 HB}

A Figura 5 apresenta os resultados da velocidade de quei$\mathrm{ma}(\mathrm{mm} / \mathrm{min})$ para os sistemas em estudo. Pode-se observar que a introdução da argila sem tratamento (AST) ao PE não alterou significativamente a velocidade de queima do PE, como já esperado, em função provavelmente da pobre interação polímero-argila. Já na presença do PE-g-MA houve uma discreta redução nesta propriedade. A incorporação do sal Cetremide aos sistemas diminuiu a velocidade de queima quando comparada aos polímeros puros (PE e PE-g-MA), provavelmente por este melhorar a interação polímero-argila, já que o comprimento do grupo alquil (16 carbonos) confere à argila o caráter orgânico necessário para aumentar a interação com os polímeros. Essa diminuição foi bastante expressiva para as composições PE/AST/Cetremide 3\% (redução de $21 \%$ em relação ao PE puro) e PE-g-MA/AST/Cetremide 3\% (redução de $26 \%$ em relação ao PE-g-MA puro). Isso pode ser atribuído à melhor interação polímero-argila, favorecida pela presença do sal e pelo grupo funcional MA presente no PE. Dessa forma, a presença da argila pode ter formado uma camada carbonácea retardando a velocidade de queima do 
material, pelo efeito de barreira aos produtos voláteis gerados durante a combustão do nanocompósito, impedindo que estes produtos escapem a alimentem a chama ${ }^{[14,16]}$. Para os sistemas com argila tratada, pode-se observar que todas as composições apresentaram redução da velocidade de queima, principalmente para o híbrido PE/PE-g-MA/ACT, o que é interessante do ponto de vista tecnológico, pois o PE-g-MA apesar de ter um custo superior em relação ao PE puro foi

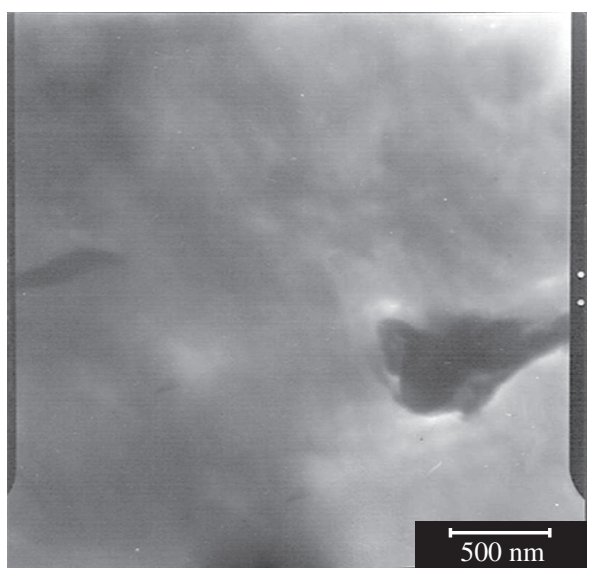

(a)

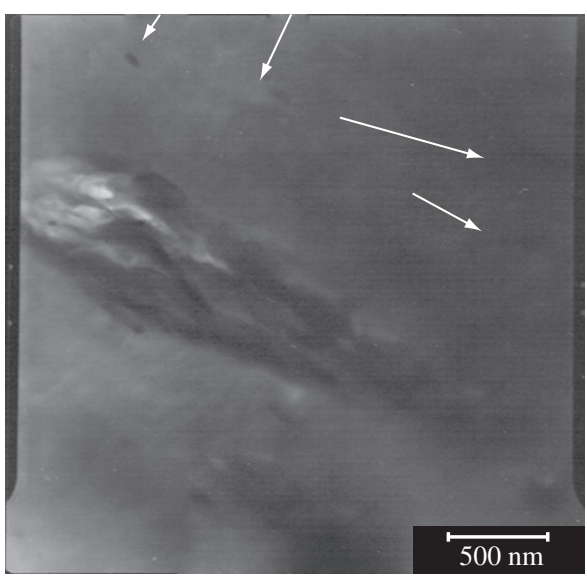

(b)

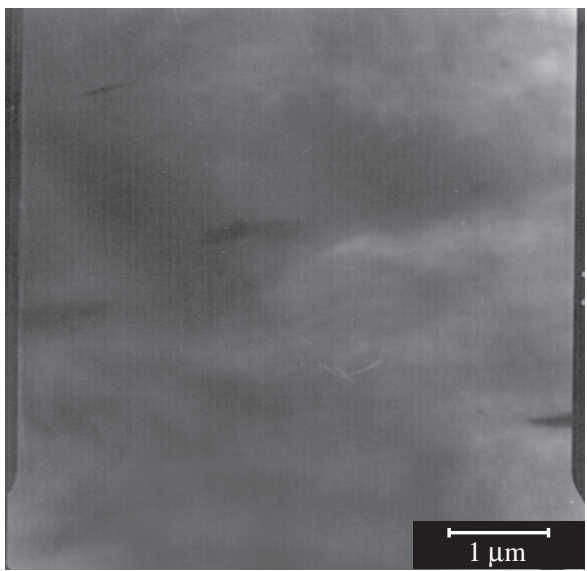

(c)

Figura 4. Fotomicrografias dos sistemas com $3 \%$ em peso de argila com tratamento: a) PE/ACT; b) PE-g-MA/ACT; e c) PE/PE-g-MA/ACT. usado com baixíssimo teor e mesmo assim alterou as propriedades do sistema. Provavelmente, um pequeno aumento no teor do graftizado e no teor de argila possa produzir propriedades marcantes nos nanocompósitos, comparadas ao sistema PE-g-MA/AST/Cetremide 3\% ou até melhor. Dos resultados apresentados para a velocidade de queima das amostras, pode-se dizer que a presença do PE graftizado e da argila é de extrema necessidade para retardar a propagação da chama, ou seja, diminuir a inflamabilidade do PE puro.

\section{Propriedades mecânicas}

A Tabela 2 mostra os resultados dos ensaios mecânicos de tração e impacto para o polietileno puro, o polietileno graftizado com anidrido maleico (PE-g-MA) puro e demais composições. Observa-se que a presença da argila e a variação do teor de sal exercem efeito considerável nas propriedades mecânicas dos materiais estudados. De acordo com a Tabela 2, as composições com polietileno apresentaram um aumento no módulo de elasticidade quando comparado com as composições com polietileno graftizado. Esse aumento foi bastante nítido para o $\mathrm{PE} / \mathrm{AST} /$ Cetremide $1 \%$. Os resultados também indicam que o módulo de elasticidade dos sistemas com argila e com a presença do compatibilizante foi superior ao do PE puro, evidenciando aumento na rigidez do nanocompósito produzido. O que corrobora com os dados acima apresentados para o teste de inflamabilidade. Novamente, o híbrido PE/PE-g-MA/ACT apresenta um aumento discreto em relação à matriz pura PE. Como mencionado acima para o teste de inflamabilidade, talvez o teor de PE-g-MA e de argila não tenha sido o suficiente para melhorar significativamente as propriedades mecânicas. Com relação à resistência à tração nota-se que não houve alterações significativas. $\mathrm{O}$ alongamento na ruptura também não apresenta grandes variações quando comparado ao PE. Os resultados obtidos para a resistência ao impacto Izod das amostras estudadas

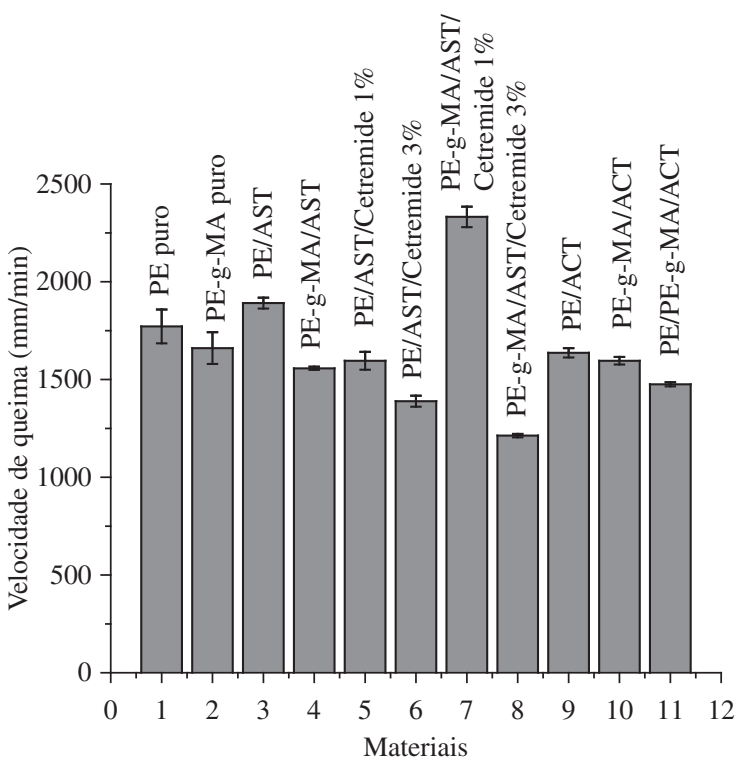

Figura 5. Velocidade de queima $(\mathrm{mm} / \mathrm{min})$ do PE puro e seus nanocompósitos. 
apresentam valores inferiores quando comparados ao PE puro, o que era de se esperar já que a presença da argila e do compatibilizante enrijeceu os sistemas. É interessante destacar que o híbrido PE/PE-g-MA/ACT alcança uma resistência ao impacto superior aos demais sistemas com um módulo também considerável. Como já comentado, esse sistema parece apresentar propriedades mecânicas e de inflamabilidade promissoras. Em geral, os sistemas polímero/argila, contendo baixo teor de argila $(<10 \%)$ freqüientemente exibem melhores propriedades mecânicas quando comparadas às propriedades do polímero puro. A razão principal para este aumento de propriedades, segundo Ray \& Okamoto ${ }^{[19]}$ e Alexandre \& Dubois ${ }^{[3]}$ é a interação interfacial mais forte entre a matriz e a camada de silicato comparada a sistemas reforçados com carga convencional.

A Figura 6 apresenta as curvas de torque em função do tempo para o PE e o PE-g-MA puros, PE e PE-g-MA com argila organofílica e o híbrido PE/PE-g-MA/ACT. Pode ser visualizado que o PE puro apresenta valor de torque maior que o PE-g-MA puro, o que era de se esperar em função dos pesos moleculares desses polímeros. O PE/ACT apresenta o valor de torque menor que todos os demais sistemas, inclusive em relação ao PE-g-MA/ACT. Isso pode ser atribuído ao fato do polietileno graftizado apresentar uma estrutura polar, havendo assim uma maior interação com a argila, tornando a estrutura mais resistente e aumentando a viscosidade. Entretanto, é interessante observar a influência da argila tratada e do PE-g-MA no sistema híbrido (PE/PE-g-MA/ACT) que apresentou um valor do torque superior aos demais. Provavelmente, a polaridade do PE-g-MA e a argila tratada promoveram uma maior eficiência de mistura com o PE do que estes mesmos componentes quando incorporados independentemente, ou seja, PE/ACT e PE-g-MA/ACT. Essa análise corrobora com os resultados acima apresentados para o teste de inflamabilidade, as propriedades mecânicas e a caracterização estrutural.

\section{Conclusões}

Nanocompósitos de polietileno de alta densidade/argila bentonita nacional foram obtidos por meio da técnica de intercalação por fusão e a influência da argila e do agente compati- bilizante PE-g-MA foi investigada por meio das propriedades morfológicas, mecânicas, reológicas e de inflamabilidade. Os resultados de difração de raios X e microscopia eletrônica de transmissão exibiram estruturas parcialmente esfoliadas e/ou intercaladas. O híbrido PE/PE-g-MA/ACT apresentou diversas partículas de argila dispersas no PE com comprimentos na faixa de $500 \mathrm{~nm}$, confirmando a intercalação das moléculas de PE entre as lamelas da argila e o sistema PE-g-MA/AST/Cetremide $3 \%$ que não apresentou pico basal exibiu uma estrutura de lamelas de argila com tendência à esfoliação. $\mathrm{O}$ teste de inflamabilidade mostrou que a adição da argila na matriz polimérica e a presença do PE graftizado retardam a velocidade de queima do sistema, ou seja, diminuem a inflamabilidade do PE puro. Quanto às propriedades mecânicas, em geral foi observado que o módulo de elasticidade das composições com polietileno foi maior do que o módulo das composições com polietileno grafitizado. O híbrido PE/PE-g-MA/ACT alcançou uma resistência ao impacto superior aos demais sistemas com um módulo também considerável, bem como, o valor do torque para esse material foi o maior obtido em relação aos demais. Aparentemente, esse sistema parece apresentar propriedades mecânicas e de inflamabilidade promissoras.

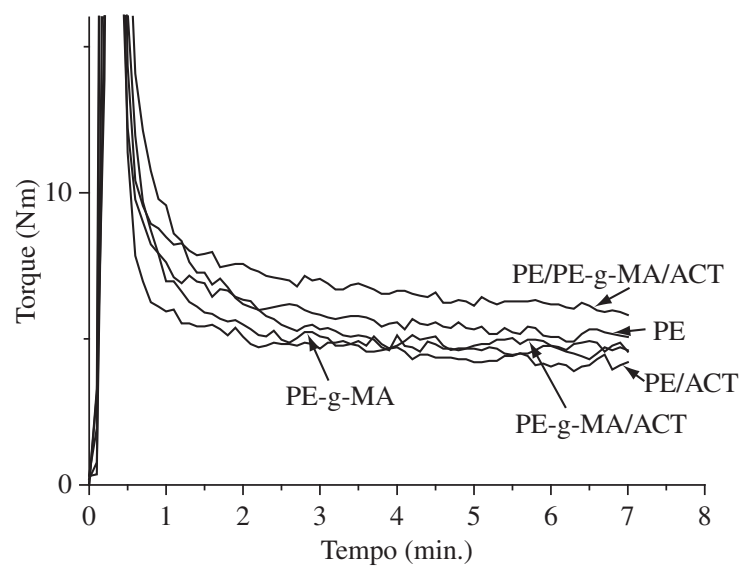

Figura 6. Caracterização reológica do PE puro e seus nanocompóstios com argila organofílica (ACT).

Tabela 2. Propriedades mecânicas do PE puro e seus nanocompósitos.

\begin{tabular}{lcccc}
\hline \multicolumn{1}{c}{ Material } & $\begin{array}{c}\text { Módulo de } \\
\text { elasticidade (MPa) }\end{array}$ & $\begin{array}{c}\text { Resistência à tração } \\
(\mathbf{M P a})\end{array}$ & $\begin{array}{c}\text { Alongamento na } \\
\text { ruptura (\%) }\end{array}$ & $\begin{array}{c}\text { Resistência } \\
\text { ao impacto (J/m) }\end{array}$ \\
\hline PE puro & $456,52 \pm 27,30$ & $22,08 \pm 0,54$ & $15,76 \pm 0,85$ & $54,12 \pm 3,77$ \\
PE-g-MA puro & $445,22 \pm 46,60$ & $21,11 \pm 0,17$ & $15,89 \pm 0,91$ & $39,55 \pm 0,55$ \\
PE/AST & $506,11 \pm 49,20$ & $22,35 \pm 0,35$ & $13,75 \pm 0,84$ & $31,63 \pm 2,02$ \\
PE-g-MA/AST & $407,36 \pm 41,30$ & $21,88 \pm 0,60$ & $17,23 \pm 1,22$ & $30,4 \pm 1,79$ \\
PE/AST/Cetremide 1\% & $675,65 \pm 68,70$ & $21,10 \pm 0,14$ & $12,75 \pm 0,58$ & $34,56 \pm 0,57$ \\
PE/AST/Cetremide 3\% & $491,88 \pm 44,50$ & $20,90 \pm 0,42$ & $16,37 \pm 0,75$ & $40,74 \pm 0,89$ \\
PE-g-MA/AST/Cetremide 1\% & $480,2 \pm 41,10$ & $22,47 \pm 0,65$ & $16,32 \pm 0,92$ & $21,93 \pm 1,02$ \\
PE-g-MA/AST/Cetremide 3\% & $420,39 \pm 19,90$ & $23,05 \pm 0,58$ & $17,61 \pm 1,02$ & $20,19 \pm 1,41$ \\
PE/ACT & $517,66 \pm 40,30$ & $22,11 \pm 0,67$ & $15,03 \pm 0,41$ & $36,23 \pm 1,10$ \\
PE-g-MA/ACT & $484,17 \pm 44,40$ & $22,70 \pm 0,39$ & $16,40 \pm 0,36$ & $21,4 \pm 1,44$ \\
PE/PE-g-MA/ACT & $473,34 \pm 38,30$ & $21,80 \pm 0,83$ & $13,44 \pm 6,05$ & $42,92 \pm 0,09$ \\
\hline
\end{tabular}




\section{Agradecimentos}

Os autores agradecem à Braskem pelo fornecimento do $\mathrm{PE}$, à Bentonit União Nordeste pelo fornecimento da argila, à Crompton/SP pelo fornecimento do PE-g-MA, à RENAMI (Rede de Nanotecnologia Molecular e de Interfaces), ao PIBIC pela bolsa de IC e ao MCT/CNPq pelo apoio financeiro.

\section{Referências Bibliográficas}

1. Yang, F.; Ou, Y. \& Yu, Z. J. - J. Appl. Polymer Sci. 69, p.355 (1998).

2. Wang, S. F.; Hu, Y.; Song, L.; Wang, Z. Z.; Chen, Z. Y. \& Fan, W. C. - Polym. Degrad. Stab., 77, p.423 (2002).

3. Alexandre, M. \& Dubois, P. - Mater. Sci. Eng., 28, p.1 (2000).

4. Gilman, J. W. - Appl. Clay Sci., 15, p.31 (1999).

5. Komarneni, S. - Journal of Mater. Chem., 2, p.1219 (1992).

6. Cho, J. W. \& Paul, D. R. - Polymer, 42, p.1083 (2000).

7. Santos, P. S. - "Ciência e tecnologia de argilas: Fundamentos", 1, 2 ed., São Paulo, Edgar Blucher (1989).

8. Barbosa, R.; Araújo, E. M.; Maia, L. F.; Pereira, O. D. \& Mélo, T. J. A. - Polímeros: Ciência e Tecnologia, 16, p.246 (2006).

9. Valenzuela-Díaz, F. R. - "Obtenção de Argilas Organofílicas Partindo-se de Argila Esmectítica e do Sal Quaternário de Amônio ARQUAD 2HT-75", in: Anais do $43^{\circ}$ Congresso Brasileiro de Cerâmica, p. 43201, Florianópolis - SC (1999).

10. Valenzuela-Díaz, F. R. - "Preparação ao Nível de Laboratório de Algumas Argilas Esmectíticas Organofilicas”, Tese de Doutorado, Escola Politécnica da Universidade de São Paulo, Brasil (1994).

11. Valenzuela-Díaz, F. R. - Key Eng. Mat., 189-191, p. 203 (2001).

12. Araújo, E. M.; Mélo, T. J. A.; Santana, L. N. L.; Neves, G. A.; Ferreira, H. C.; Lira, H. L.; Carvalho, L. H.; Ávvila Jr., M. M.;
Pontes, M. K. G. \& Araújo, I. S. - Mat. Sci. and Eng. B, 112, p.175 (2004).

13. Vieira José, C. L.; Pinto, C. A.; Valenzuela-Díaz, F. R. \& Buchler, P. M. - "Sintetização de Argilas Organofílicas Visando seu Uso no Controlo Ambiental de Resíduos de Fenol", in: Anais do $46^{\circ}$ Congresso Brasileiro de Cerâmica, p.1, São Paulo - SP (2002).

14. Barbosa, R. - "Efeito de sais quaternários de amônio na organofilização de uma argila bentonita nacional para o desenvolvimento de nanocompósitos de polietileno de alta densidade (PEAD)", Dissertação de Mestrado, Universidade Federal de Campina Grande, Brasil (2005).

15. Araújo, E. M.; Oliveira, A. D.; Araújo, H. L. D.; Araújo, K. D.; Barbosa, R. \& Mélo, T. J. A. - Polímeros: Ciência e Tecnologia, 16, p.38 (2006).

16. Barbosa, R.; Araújo, E. M; Melo, T. J. A. \& Ito, E. N. - Mat. Letters, 61, p.2575 (2007).

17. Araújo, E. M.; Barbosa, R.; Rodrigues, A. W. B. \& Ito, E. N. Mat. Sci. and Eng. A, 445-446, p.141 (2007).

18. Kornmann, X. - "Synthesis and Characterization of Thermoset - clay Nanocomposites", Introduction, Lulea Tekniska Universite (1999).

19. Ray, S. S. \& Okamoto, M. - Progress in Polym. Sci., 28, p.1539 (2003).

20. Barbosa, R.; Araújo, E. M; Melo, T. J. A \& Ito, E. N. - Polímeros: Ciência e Tecnologia, 17, nº 2, p.104 (2007).

21. UL-94: Test for Flammability of Plastic Materials for Parts in Devices and Appliances. Underwriters Laboratories Inc (UL), (2001).

22. Zanetti, M. \& Costa, L., Polymer, 45, p.4367 (2004).

23. Paiva, L. B.; Morales, A. R. \& Guimarães, T. R. - Polímeros: Ciência e Tecnologia, 16, p.136 (2006).

Enviado: $28 / 06 / 07$ Reenviado: 10/12/07

Aceito 15/12/07 